\title{
La fable du Martien, de Max et d'Alfred Weber : une introduction au paradoxe fondamental de l'économie spatiale
}

\author{
Luc-Normand Tellier \\ Université du Québec à Montréal
}

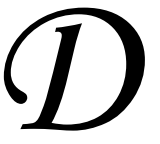

ans mon récent ouvrage ${ }^{1}$, plusieurs pages sont consacrées à la compréhension de ce que j'appelle le paradoxe fondamental de l'économie spatiale. Afin de faire découvrir ce paradoxe, j'avais imaginé une fable que nous avons dû renoncer à inclure dans le livre, faute d'espace.

Ce conte philosophique raconte la rencontre imaginaire entre un Martien et deux frères célèbres : Max Weber (1864-1920), économiste, sociologue et philosophe allemand connu, entre autres, pour le lien qu'il a tenté d'établir entre le protestantisme et le capitalisme, et Alfred Weber (1868-1958), un des grands penseurs de l'économie spatiale. La fable se présente comme suit.

Il était une fois, au début de 1920, un Martien qui survolait la planète Terre. Après avoir observé les lumières qui parsemaient la surface de la terre la nuit, il décida de se poser. Le hasard voulut qu'il atterrisse à Munich en Allemagne, un dimanche matin, dans le jardin du professeur Max Weber qui recevait alors la visite de son frère Alfred. Max avait 55 ans et il enseignait à l'Université de Munich, tandis qu'Alfred, 51 ans, était rattaché à l'Université d'Heidelberg. Tous deux s'intéressaient au phénomène urbain; ils avaient même écrit des livres là-dessus ${ }^{2}$. L'un et l'autre se considéraient comme sociologues, quoique Alfred ait eu un

$$
\begin{gathered}
\text { Il était une fois, au début de } \\
\text { 1920, un Martien qui survolait } \\
\text { la planète Terre. }
\end{gathered}
$$

Heureusement, le Martien descendu de sa soucoupe volante parlait parfaitement l'allemand (cette capacité d'apprendre instantanément les langues étrangères n'est-elle pas une preuve de la supériorité de l'intelligence des Martiens sur celle des humains?). "Bonjour, dit le Martien aux frères Weber, enchanté de faire votre connaissance. L'on m'a dit que vous étiez deux des habitants penchant beaucoup plus marqué pour la science économique, en particulier pour l'économie spatiale.

Max était déjà célèbre. Ses livres et ses articles soulignaient l'importance de la culture et de la religion dans le développement économique. Sa thèse la plus célèbre avançait que le protestantisme avait joué un rôle majeur dans l'apparition et le développement du les plus intelligents de cette planète. Peut-être pourriez-vous $m$ 'expliquer comment il se fait que les Terriens se comportent d'une façon qui me semble complètement illogique? "Max prit la parole et répondit: "Nous vous souhaitons la bienvenue, Monsieur. le Martien. Nous, Terriens, sommes à l'occasion émotifs, superstitieux irrationnels et même un peu fous; cependant, tout compte fait, nous prétendons être 
rationnels, logiques, pleins de bon sens, et même sages. Je m'étonne que vous ayez l'impression que nous ne le sommes pas. Tout d'abord, dites-moi, depuis combien de temps nous observez-vous? Peut-être votre jugement est-il trop hâtif? " Le Martien répliqua : "Mon groupe et moi-même survolons et observons la Terre depuis déjà quelques années. Cependant, ce n'est que cette semaine que nous avons osé atterrir pour la première fois. Nous n'avons rencontré jusqu'ici que quelques personnes. La plupart de celles que nous avons interrogées nous ont laissé entendre que vous êtes, parmi les Terriens, de ceux qui pourraient le mieux nous éclairer sur le comportement et l'intelligence relative des humains." "Mais, que cherchez-vous à comprendre précisément? », demanda Alfred.

"Tout d'abord, répondit le Martien, vue du haut des airs, votre planète semble peu habitée, si ce n'est par des animaux dépourvus d'intelligence. Nous avons vu de vastes déserts inhabités, d'immenses océans sur lesquels il n'y avait presque personne, des régions polaires désolées et presque vides, des chaînes de montagnes et de denses forêts avec très peu d'habitations et encore moins de villages ou de villes. "

"Suivant nos calculs, votre planète est couverte d'eau à 70,8\% et de glace à $3 \%$. Cela veut dire que les terres représentent seulement 26,2\% de la superficie totale de 510065000 kilomètres carrés de cette planète. Vous remarquerez que nous maîtrisons déjà parfaitement votre système d'unités de mesure... Or, si l'on enlève de la superficie occupée par les terres les zones relativement inhabitées pour ne garder que les terres arables, les forêts et les steppes où nous avons repéré des populations denses ou clairsemées, il ne reste que 68200 kilomètres carrés, soit $2 \%$ de la superficie totale de la planète ».

«En somme, les Terriens n'habitent vraiment que $2 \%$ de la superficie de leur planète, ce qui nous pousse à nous interroger grandement sur leur niveau d'intelligence. Bien plus, nous avons remarqué que les steppes, qui représentent 0,41\% de la superficie totale de la planète et les forêts, qui représentent $0,59 \%$ de cette même superficie, sont beaucoup moins habitées que les terres arables. Or, ces dernières ne représentent que $1 \%$ de la superficie totale de votre globe. Comment ne pas s'interroger sur le niveau d'intelligence d'une espèce qui ne réussit qu'à occuper de façon systématique que $1 \%$ de l'espace disponible sur sa planète?"

"Mais là ne s'arrête pas notre étonnement, nous avons examiné de plus près les terres arables et nous avons été sidérés de constater que ces dernières sont occupées de façon tout à fait inégale. Ainsi, $90 \%$ des Terriens vivent dans l'hémisphère nord et seulement $10 \%$, dans l'hémisphère sud. Dans toutes les zones habitées, nous avons repéré des vallées et des plaines où les maisons et les villages étaient tout à fait éparpillés. Puis nous avons remarqué que les Terriens semblent aimer l'eau. On les retrouve en beaucoup plus grand nombre sur les côtes et le long des rivières. Enfin, en descendant à plus basse altitude, nous avons constaté qu'il existait, ici et là, d'immenses concentrations de population occupant une partie infime, pour ne pas dire microscopique, de la planète. Ces concentrations, que vous appelez des métropoles, portent des noms étranges: Tokyo, Paris, Londres, Berlin, New York, Istanbul, Bagdad, Le Caire, Bombay, Pékin, Shanghai, Lagos... et elles nous semblent incompréhensibles. Suivant notre enquête, les Terriens y vivent dans des conditions affreuses marquées par la pauvreté, le bruit, la pollution, la saleté, le crime, la maladie, l'insécurité, le stress, l'agressivité, le surmenage, etc., alors même que l'espace ne manque pas sur $99 \%$ de votre planète et même sur ces terres arables que vous semblez tant apprécier. »

\section{« En somme, les Terriens n'habitent vraiment que $2 \%$ de la superficie de leur planète, ce qui nous pousse à nous interroger grandement sur leur niveau d'intelligence. »}

"Si les Terriens sont intelligents, pourquoi, diable (nous avons appris que vous croyiez au diable; étrange! nous n'en avons vu aucun...), pourquoi se répartissent-ils de cette manière sur cette planète? Nous avons même noté qu'existaient des regroupements de villes et que les métropoles sont généralement entourées d'un grand nombre de villes moyennes et que ces dernières sont aussi entourées de petites villes. En somme, il existe des grappes de villes très nettement visibles du haut des airs. À première vue, cela nous semble tout à fait étrange et difficilement compréhensible. Pourquoi ne pas occuper tout l'espace disponible de façon équilibrée plutôt que de s'entêter à vous 
empiler les uns sur les autres dans des espaces déjà congestionnés et surchargés? Pourquoi vous concentrer là où vous l'êtes déjà et vider les espaces qui sont à peu près vides? Expliquez-nous. »

Les deux frères Weber se regardèrent, interloqués. Oui, à bien y penser, cela leur semblait constituer des interrogations tout à fait légitimes et pleines de bon sens. Oui, ils comprenaient tout à coup que le Martien pouvait s'interroger sur la rationalité des Terriens. Cependant, quelque chose au fond d'euxmêmes les empêchait d'avouer au Martien leur réaction profonde. Il fallait sauver l'honneur de la race humaine et défendre à tout prix sa rationalité. Une contre-attaque fut improvisée.

Alfred risqua une première réplique: "Oui, vous avez raison, tout cela peut sembler illogique. Mais ça ne l'est pas. Tout d'abord, comme mon frère l'a si bien souligné dans ses livres, nous, Terriens, aimons nous côtoyer les uns les autres; nous aimons être ensemble, vivre en société, suivant notre expression. Il y a là une part de l'explication. Mais il y a aussi d'autres facteurs qui jouent. Nous déplacer d'un point à un autre sur Terre implique des coûts, entre autres à cause de la gravité, qui est beaucoup plus forte ici que sur la lune; par exemple, ce qui pèse 100 newtons sur la Terre ne pèse que 16,3 newtons sur la lune. On fait d'ailleurs allusion à ces cô̂ts de déplacement ou de transport lorsque nous parlons de la "friction de l'espace". Je pourrais vous démontrer mathématiquement que, strictement du point de vue des cô̂ts de transport, le regroupement des activités et des populations se justifie rationnellement. "

Le Martien répliqua : "Si ce que vous dites est vrai et si le regroupement est si logique, comment se fait-il que, selon nos observations et nos propres calculs, en cette année 1920, moins de $3 \%$ de la population mondiale se retrouve dans les grandes villes et environ $12 \%$, dans les petites ou moyennes villes, alors qu'au moins $85 \%$ de la population vit à la campagne? Si le fait de se regrouper est logique, c'est dire qu'au moins $85 \%$ des Terriens sont illogiques... » Cette réplique du Martien laissa Alfred bouche bée.

Son frère Max vint à la rescousse. "Monsieur le Martien, dit-il, nous, Terriens, aimons vivre ensemble, mais nous aimons aussi la solitude et la liberté que procure la vie à la campagne. Et, s'il est vrai qu'il y a des raisons économiques qui justifient le re- groupement des activités et des populations, il y a aussi d'excellentes raisons économiques d'éparpiller certaines activités comme l'agriculture, l'élevage, la coupe de bois, etc. " Le Martien intervint : "Je crois que je commence à comprendre. Il y a ceux qui aiment se regrouper ou qui sont forcés de le faire et il y a ceux qui aiment vivre à l'écart ou qui sont forcés de le faire. C'est sans doute pour cela que nous avons repéré deux types de lumières dans la nuit: les lumières qui bougent et les lumières qui ne bougent pas. Je suppose que les lumières qui ne bougent pas sont celles des gens qui sont établis soit à la ville, soit à la campagne, tandis que les lumières qui bougent sont celles des véhicules qui transportent des personnes ou des biens des lieux d'habitation éparpillés vers les villes et les villages, et vice versa, ou encore des concentrations de populations et d'activités vers d'autres lieux de concentration. Ainsi, par le biais du transport, les uns et les autres se complètent tout en se conformant à leurs préférences et à leurs contraintes respectives. " "Voilà! Vous comprenez! », s'exclama Max.

\section{« Pourquoi vous concentrer là où vous l'êtes déjà et vider les espaces qui sont à peu près vides ? 》}

Alfred enchaîna: "De fait, ce que vous venez de dire est hautement important. Afin de comprendre la distribution spatiale des lumières qui ne bougent pas, vous devez comprendre la logique du transport, c'està-dire la logique des lumières qui bougent. Inversement, si vous cherchez à comprendre la logique des lumières qui bougent et la répartition des flux de lumières entre les diverses concentrations de lumières qui ne bougent pas, vous devez comprendre la logique qui préside à la répartition spatiale des lumières qui ne bougent pas. De fait, les coûts de déplacement expliquent la distribution spatiale des localisations fixes, tandis que les coûts immobiliers, à savoir les coûts de l'immobilité, expliquent la répartition spatiale des flux. En somme, le coût de la mobilité explique l'immobilité et le coût de l'immobilité explique la mobilité. Mobilité et immobilité sont indissociables. Ils s'expliquent l'un l'autre. Ils sont comme le reflet l'un de l'autre dans le miroir. "

Impressionné, le Martien s'exclama : "Tout cela devient aussi abstrait que la philosophie allemande..." 
Après un long moment de silence, le Martien reprit le dialogue: "Si certains Terriens aiment vivre regroupés et d'autres préfèrent l'isolement, comment se faitil que certains Terriens se retrouvent dans de grandes villes et d'autres, dans de petites ou moyennes villes? N'existe-t-il pas une taille optimale de ville où tous les Terriens qui aiment vivre en ville pourraient se retrouver?»

Pris au dépourvu, Max et Alfred se regardèrent. Max prit l'initiative et répondit: "Vous avez raison. À mon avis, nous, citoyens, devrions tous vivre dans des villes de taille moyenne. Je considère que, des points de vue politique, social, culturel et simplement hédoniste, les grosses villes sont monstrueuses, tandis que les petites villes sont ennuyeuses au possible. Logiquement, les gouvernements devraient réglementer la taille des villes et exiger que toutes les villes aient la taille optimale. Je ne vois d'ailleurs pas pourquoi ils ne le font pas. "

Alfred protesta. "Mais, Max, tu sais bien qu'ils ne le pourraient pas. Il n'y a pas un gouvernement sur Terre qui pourrait interdire les petites villes et forcer les grosses villes à réduire leur population. Même les gouvernements les plus dictatoriaux n'y arriveraient pas, du moins sur une longue période de temps. Ils ne le pourraient pas et, quant à moi, ils ne le devraient pas tout simplement parce qu'il y a de bonnes raisons économiques d'avoir des villes de plusieurs tailles différentes. Les villes forment des familles ou ce que nous appelons des systèmes urbains. Comme dans toutes les familles, il y a les grands et les petits, les adultes et les jeunes, les leaders et les soumis, les forts et les protégés. Une grande ville offre des biens et des services que des villes plus petites ne peuvent pas offrir, et les plus petites villes offrent des biens et des services aux populations environnantes qui souhaitent éviter de se rendre dans les grandes villes situées plus loin. Les grandes villes ont besoin des petites villes pour étendre leur influence sur les territoires plus éloignés, et les petites villes ne sauraient survivre sans l'apport des grandes villes. En somme, nous avons besoin à la fois de grandes et de petites villes. Les grandes villes et les petites villes ont besoin les unes des autres, comme les membres d'une même famille ont besoin les uns des autres. Les flux de personnes et de biens que vous observez autour des villes sont la preuve que tous les éléments du système apportent quelque chose aux autres parties du système. Et plus une ville est grosse, plus vous verrez que les flux qui l'alimentent et qui en sortent sont importants. Là aussi, au niveau du système urbain, les éléments immobiles du système déterminent l'ampleur des mouvements, tout comme les prix attachés aux déplacements des personnes et des biens déterminent l'univers des localisations. "

De plus en plus intéressé, le Martien enchaîna: "Lors de notre courte enquête, on nous a dit que même les localisations ont un prix. Les Terriens sont prêts à payer pour s'immobiliser, s'établir à certains endroits plutôt qu'à d'autres. Cela nous apparaît étrange. Pourquoi payer pour rester à un endroit plutôt qu'à un autre? Mais le plus étrange, c'est que, selon notre recherche, les Terriens qui paient le plus cher pour les terrains qu'ils occupent sont précisément ceux dont les terrains sont souvent situés dans les zones les plus congestionnées, les plus bruyantes, les plus dangereuses et les plus polluées. Cela est d'autant plus troublant qu'on nous dit que les Terriens aiment le calme, la sécurité, l'air pur et la paix. Tout cela me semble bien illogique. "À ce point de la conversation, Max et Alfred se surprirent à penser que, peut-être, le Martien avait raison et que les Terriens devraient être honteux de se comporter de la sorte. Et s'ils n'étaient pas, après tout, aussi intelligents qu'ils le prétendaient...

\section{« Logiquement, les gouvernements devraient réglementer la taille des villes et exiger que toutes les villes aient la taille optimale. Je ne vois d'ailleurs pas pourquoi ils ne le font pas. »}

Les deux frères eurent un sursaut d'orgueil et tentèrent une nouvelle contre-offensive. Comme Max n'arrivait pas à trouver une explication sociale au phénomène souligné par le Martien, une fois de plus, Alfred intervint : "De fait, il n'y a au fond qu'un seul phénomène fondamental dans tout ce que vous décrivez. $C$ 'est celui de la polarisation. Dans une large mesure, les conditions de la mobilité sont telles que certaines localisations sont meilleures que d'autres, et certaines autres, encore meilleures que les très bonnes. Toutes les localisations ne sont pas nées égales, si je puis dire. Certaines sont destinées à être convoitées et d'autres à être négligées ou même ignorées. Cela s'explique par les conditions de mobilité, mais aussi par d'autres facteurs comme la nature des sols, les 
pentes, les paysages, l'ensoleillement, le climat, la pluviométrie, etc. »

"À cause de tous ces facteurs, la polarisation existe et elle existe à des degrés différents d'un endroit à l'autre. Or, plus une localisation est convoitée, plus son prix est élevé. Les espaces surpeuplés dont les prix sont élevés ne sont pas recherchés parce qu'ils sont surpeuplés et encore moins parce que leur prix est élevé. Ils le sont pour d'autres raisons qui expliquent qu'ils sont recherchés et chers. Cependant, la chose la plus étrange, selon moi, ce n'est pas la polarisation simple, mais c'est la polarisation des pôles. $C$ 'est le fait que des hiérarchies se forment entre les centres de polarisation, qu'un centre des centres apparaisse, qu'un centre des centres de centres émerge aussi à tel point qu'aujourd'hui, Londres est au centre de la planète tout entière. Voilà la chose la plus étrange. "

"Néanmoins, quand on y songe vraiment, tout est au fond très logique. Comme la gravité est forte sur Ter$r e$, se déplacer coûte quelque chose, d'autant plus que le relief de notre planète peut être, par endroits, très accidenté. Comme se déplacer coûte quelque chose et se déplacer ne coûte pas la même chose dans toutes les directions, quand il s'agit de choisir un emplacement en fonction de certains points avec lesquels nous voulons être régulièrement en contact, toutes les localisations ne sont pas équivalentes. Certaines localisations sont meilleures que les autres; et ce, du simple point de vue du transport, même en ignorant les autres facteurs comme la nature des sols, le climat, etc. Or, si toutes les localisations ne sont pas équivalentes, la polarisation devient naturelle, bien que son niveau puisse varier considérablement. »

\section{« Si la polarisation est naturelle à petite échelle, à l'échelle du village par exemple, elle l'est tout autant, quand on y pense bien, à des échelles plus grandes, $y$ compris à l'échelle mondiale. »}

"Il y a un siècle, en 1820, le taux d'urbanisation mondial était plus près de $5 \%$ que des $15 \%$ d'aujourd'hui, et certains annoncent même qu'il pourrait dépasser les $50 \%$ d'ici un siècle, vers 2020. De fait, la polarisation existe depuis que l'homme a commencé à se sédentariser, au néolithique, il y a 13000 ans, bien qu'au début elle ait été très, très timide. Depuis ce jour, on peut dire que, bien qu'elle ait régressé occasionnellement dans certaines régions (par exemple, au lendemain de la chute de l'Empire romain d'Occident), la polarisation n'a fait qu'augmenter, très lentement pendant des millénaires, puis soudainement, depuis un siècle, à vive allure. "

"Si la polarisation est naturelle à petite échelle, à l'échelle du village par exemple, elle l'est tout autant, quand on y pense bien, à des échelles plus grandes, y compris à l'échelle mondiale. Les conditions de la mobilité déterminent les conditions de l'immobilité et vice versa, avons-nous dit. Il découle de cela que si les conditions de la mobilité provoquent la polarisation quelque part, elles entraînent aussi la polarisation dans l'ensemble du système soumis à ces mêmes conditions. Telle est notre situation sur Terre, Monsieur le Martien. La condition humaine est née dans un contexte marqué par la gravité terrestre et elle ne s'en dégagera jamais. En dépit de toutes nos découvertes et en dépit de toutes nos inventions, nous, êtres humains, sommes condamnés à vivre avec la polarisation. "

"Chaque fois, et c'est arrivé très souvent, que nous avons cru pouvoir nous affranchir de la gravité et de la polarisation pour quitter nos villes et aller vivre au milieu de la nature entourés d'oiseaux, d'eau pure et d'air vivifiant, nous avons découvert, après quelque temps, que le monde que nous venions d'engendrer était encore plus polarisé, surpeuplé, congestionné, pollué et stressé qu'avant. C'est ce qui s'est produit après l'invention de la roue et du chariot, et c'est aussi ce qui s'est produit de notre vivant, à mon frère et à moi-même, avec l'arrivée du chemin de fer, du télégraphe, du téléphone, de la radio, de l'automobile, du camion, de l'autobus, de l'aérostat, de l'avion, du tramway et du métro. Plus nous tentons de nous affranchir de la gravité et de la polarisation par le biais d'innovations techniques, plus nous nous retrouvons asservis à la gravité et à la polarisation. Voilà, semble-t-il, notre fatum, notre fatalité à nous, pauvres Terriens. »

Max et le Martien écoutaient Alfred en silence. Ils étaient émus. Le plus émus des deux était Max, que le discours d'Alfred avait ébranlé. Non seulement Alfred faisait-il état des limitations de la nature humaine et de la condition terrestre, mais il disait que la culture et les facteurs sociaux étaient eux-mêmes soumis à la 
rigidité des contraintes terrestres. Le rêve que Max avait caressé toute sa vie de voir l'esprit de ses frères et sœurs humains dominer le monde était ébranlé. "Non, se disait Max, si ce que dit Alfred est vrai, quoi que nous fassions, ce rêve ne pourra jamais complètement se réaliser. "

Max mourut quelques mois plus tard, le 14 juin 1920 , dans sa résidence de Munich où le Martien lui avait rendu visite. Ce dernier avait alors repris le chemin de sa propre planète. Quant à Alfred, il vécut encore longtemps. Il mourut à Heidelberg 38 ans plus tard, en 1958, à l'âge vénérable de 90 ans, réconcilié avec la condition d'humain et le monde sur-polarisé de l'après-guerre.

En termes scientifiques, le « paradoxe fondamental de l'économie spatiale » réside dans le fait qu'alors que la friction de l'espace est une condition nécessaire à l'apparition de la polarisation, tout indique que plus la friction de l'espace baisse (et, par conséquent, les forces d'attraction baissent), plus la polarisation augmente et plus les forces de répulsion équilibrantes se multiplient ${ }^{3}$. Dans Tellier ${ }^{4}$, le lecteur trouvera sept explications possibles du paradoxe fondamental de l'économie spatiale.

\section{Notes et références}

1 Tellier, L.-N. (2005), Redécouvrir l'histoire mondiale, sa dynamique économique, ses villes et sa géographie, Montréal, Éditions LIBER, 592 p.

2 Weber, A. (1909), Alfred Weber's Theory of the Location of Industries, C.J. Friedrich (traducteur), Chicago, U. of Chicago Press, 1957; Weber, M. (1904-1905), L'éthique protestante et l'esprit du capitalisme, Paris, Gallimard, 2004, 341 p.; Weber, M. (1921), Économie et société, Paris, Pocket, 2003.

3

Pour mieux comprendre les concepts de forces d'attraction et de répulsion, voir Tellier, L.-N. (1993), Économie spatiale: rationalité économique de l'espace habité (seconde édition revue, augmentée et corrigée), Montréal, Éditions Gaëtan Morin, 285 p.

4 Tellier (2005), op. cit., p. 80-88. 